\title{
NOTE ON THE KINEMATICS OF PLANE VISCOUS MOTION*
}

By J. L. SYNGE (Dublin Institute for Advanced Studies)

In 1911 G. Hamel (Göttinger Nachrichten, Math.-Phys. Kl. 1911, 261-270) obtained an interesting result, which may be stated as follows:

Let $R$ be a finite plane region with boundary $B$. Then the equation $\Delta \psi=F$ possesses a solution $\psi$ for which both $\psi$ and $\partial \psi / \partial n$ vanish on $B$ if and only if $F$ satisfies

$$
\int F U d x d y=0
$$

$U$ being an arbitrary harmonic function.

In other words, for the existence of a solution with this double boundary condition, it is necessary and sufficient that the function $F$ be orthogonal to the linear space of harmonic functions.

The hydrodynamical interpretation of Hamel's theorem is as follows. For an incompressible fluid moving in the plane with vorticity $\omega$, we have

$$
u_{x}+v_{y}=0, \quad v_{x}-u_{\nu}=2 \omega,
$$

and there is a stream-function $\psi$ such that

$$
u=-\psi_{y}, \quad v=\psi_{x}, \quad \Delta \psi=2 \omega .
$$

Thus Hamel's theorem tells us that in order that a given distribution of vorticity may be consistent with vanishing velocity on the boundary $B$ (the usual boundary condition for a viscous fluid in a fixed container), it is necessary and sufficient that

$$
\int \omega U d x d y=0
$$

$U$ being an arbitrary harmonic function.

However, inspection of Hamel's proof (loc. cit. p. 266) shows that he made use of a Green's function of the second type, i.e. a harmonic function $G_{2}$ with a singularity $\log r$ and making $\partial G_{2} / \partial n=0$ on $B$. There is, of course, no such function for Laplace's equation, since this singularity and this boundary condition are inconsistent.

Not knowing of Hamel's work, I obtained Hamel's result in 1935 in a rather special case (Proc. London Math. Soc. 40 (1935), 23-36) in a different way.** In the present note the theorem is extended to include compressibility.

Theorem: A compressible viscous fluid moves inside a fixed connected boundary $B$, on which the velocity vanishes. An expansion $\theta(x, y)$ and a vorticity $\omega(x, y)$ are consistent with this boundary condition if, and only if,

$$
\int(2 \omega U-\theta V) d x d y=0,
$$

${ }^{*}$ Received November 16, 1949.

${ }^{* *}$ Footnote added in proof $(F e b .20,1950)$ : The result (4) has recently been proved by J. Kampé de Fériet (Math. Mag. 21, 71-79(1947); Ann. Soc. Sci. Bruxelles (I) 62, 11-18(1948). 
where $U$ is an arbitrary harmonic function and $V$ the conjugate harmonic function, such that

$$
U_{x}=V_{y}, \quad U_{\nu}=-V_{x} .
$$

In purely mathematical language, equation (5) is a necessary and sufficient condition for the consistency of the equations

$$
u_{x}+v_{y}=\theta, \quad v_{x}-u_{y}=2 \omega, \quad(u)_{B}=0, \quad(v)_{B}=0 .
$$

Proof: Let $l, m$ be the direction cosines of the outward normal to $B$. Let $\theta$ and $\omega$ be arbitrarily assigned. Let $u^{\prime}, v^{\prime}$ satisfy

$$
u_{x}^{\prime}+v_{\nu}^{\prime}=\theta, \quad v_{x}^{\prime}-u_{y}^{\prime}=2 \omega, \quad\left(l u^{\prime}+m v^{\prime}\right)_{B}=0 .
$$

It is well known that the solution $\left(u^{\prime}, v^{\prime}\right)$ is unique, since the two partial differential equations define $\left(u^{\prime}, v^{\prime}\right)$ to within the gradient of a harmonic function, and the normal derivative of the latter on $B$ is then given by the last of (8).

Denoting the integral in (5) by $I$, we have

$$
\begin{aligned}
I= & \int(2 \omega U-\theta V) d x d y \\
= & \int\left[U\left(v_{x}^{\prime}-u_{\nu}^{\prime}\right)-V\left(u_{x}^{\prime}+v_{y}^{\prime}\right)\right] d x d y \\
= & \int\left[u^{\prime}\left(U_{\nu}+V_{x}\right)+v^{\prime}\left(V_{\nu}-U_{x}\right)\right] d x d y \\
& \quad+\int_{B}\left[U\left(l v^{\prime}-m u^{\prime}\right)-V\left(l u^{\prime}+m v^{\prime}\right)\right] d s
\end{aligned}
$$

or, by (6) and (8),

$$
I=\int_{B} U\left(b v^{\prime}-m u^{\prime}\right) d s .
$$

Now if $I=0$ for arbitrary harmonic $U$, it follows that

$$
\left(l v^{\prime}-m u^{\prime}\right)_{B}=0,
$$

since the values of $U$ on $B$ may be arbitrarily assigned. Combining (8) with (11) we get $\left(u^{\prime}\right)_{B}=0,\left(v^{\prime}\right)_{B}=0$; thus the condition $I=0$ is sufficient.

On the other hand, if (7) are consistent, then $\left(u^{\prime}\right)_{B}=0,\left(v^{\prime}\right)_{B}=0$, and so, by (10), $I=0$; thus the condition $I=0$ is necessary.

We get Hamel's theorem on putting $\theta=0$. 